Chin. J. Astron. Astrophys. Vol. 0, No. 0, (200x) 000-000

(http://www.chjaa.org)

Chinese Journal of

Astronomy and

Astrophysics

\title{
Fireball and Cannonball Models of Gamma-Ray Bursts Confront Observations
}

\author{
Arnon Dar \\ Physics Department and Space Research Institute \\ Technion - Israel Institute of Technology, Haifa 32000, Israel \\ Received 2005 September 27; accepted 2005 month day
}

\begin{abstract}
The two leading contenders for the theory of gamma-ray bursts (GRBs) and their afterglows, the Fireball and Cannonball models, are compared and their predictions are confronted, within space limitations, with key observations, including recent observations with SWIFT.
\end{abstract}

\section{INTRODUCTION}

The currently best-studied theories of Gamma-Ray Bursts (GRBs), X-Ray Flares (XRFs) and their afterglows (AGs) are the Fireball (FB) models (see, e.g. Zhang \& Mészáros 2004 and Piran 2005] for recent reviews) and the Cannonball (CB) model (see, e.g., Dar \& De Rújula 20002004 Dado, Dar \& De Rújula 20022003 and references therein). In spite of their similarly sounding names, these two models are (or were initially) completely different in their basic hypothesis, in their description of the data, and in their predictions. While FB models are/were generally welcomed by the GRB community (see however, Dermer 2002), the CB model was not. In fact, many underlying ideas of the CB model, which were proved to be correct and even path-breaking for the GRB field, met skepticism, strong opposition, and initial dismissal. These ideas included the collimated nature of GRBs/XRFs and their afterglows, the SN/XRF-GRB association and the trivial unification of GRBs and XRFs, which were all suggested in print long before they were adapted in the FB models. GRBs/XRFs and their AGs are notorious for their diversity. Yet, both models claim to predict correctly their main properties. No doubt, both models are a simplification of a very complex phenomenon and may require modifications when compared with future observations. But their assumptions and predictions should be falsifiable and should be tested not against prejudices of astrophysicists, but against key observations, as I shall try to do here within space limitations.

\section{THE FB AND CB MODELS}

The current FB models of GRBs evolved a long way from the first spherical FB models, suggested by Paczynski (1986) and Goodman $(\underline{1986})^{1}$. The most popular ones (see e.g. Zhang \&

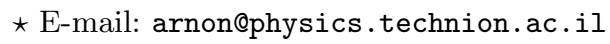

1 See, e.g., Shemi \& Piran [1990] Narayan, Paczynski \& Piran 1992] Mészáros \& Rees 1992 Mészáros \& Rees 1993 Paczynski \& Rhoads 1993 Katz 1994a1994b Mészáros \& Rees 1997
} 


\section{GRB FIREBALL MODEL}

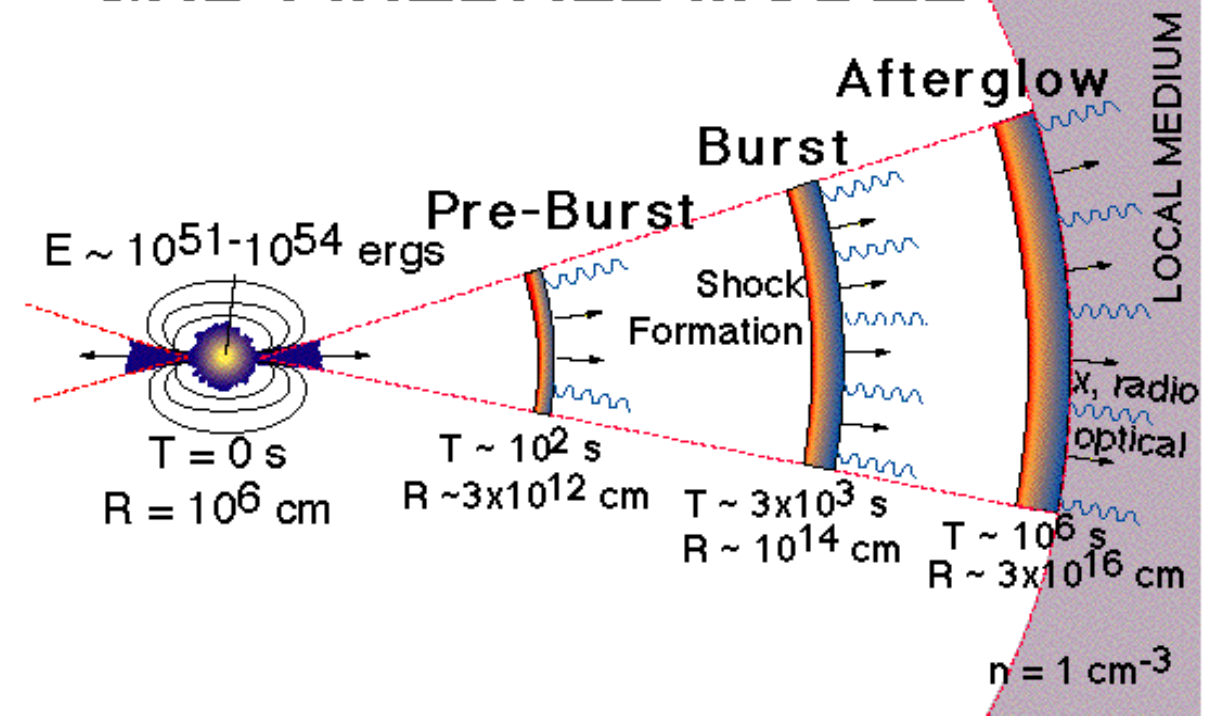

Fig. 1 Illustration of the FB model and its internal/external shock scenario, adapted from Ghisellini 2001

Mészáros 2004] and Piran 2005) assume that GRBs are emitted from highly relativistic conical fireballs (Mészáros \& Rees 1992, Levinson \& Eichler 1993. Woosley 1993a, 1993b) produced in hypernovae - an hypothesized rare class of superenergetic supernovae (SNe) type Ic, generated by direct collapse of massive stars to black holes (Paczynski 1988. Iwamoto et al. 1998, MacFadyen \& Woosley $(1999)^{2}$. The GRB pulses are assumed to be produced by synchrotron emission from collisions between highly relativistic conical shells ejected in the hypernova explosion, while the GRB afterglow is assumed to be synchrotron radiation emitted when the merged shells collide with the interstellar medium (ISM) and drive a forward blast wave into the ISM and a reverse shock into the merged shells. The FB model is illustrated in Fig. 1 1 adapted from Ghisellini 2001.

The CB model (Dar \& De Rújula 2000 2004 Dado, Dar \& De Rújula 2002 2003) is an elaboration on the ideas of De Rújula (1987), Shaviv and Dar (1995), Dar (1997, 1998) and Dar \& Plaga (1999). In the CB model, long-duration GRBs and their AGs are produced by bipolar jets of CBs which are ejected in ordinary core-collapse supernova explosions. An accretion disk or torus is hypothesized to be produced around the newly formed compact object, either by stellar material originally close to the surface of the imploding core and left behind by

Waxman 1997a 1997b Dermer \& Mitman 1999] and the more recent reviews by Piran (1999 2000), Mészáros (2002), Hurley, Sari \& Djorgovski (2002), Waxman (2003a), Zhang \& Mészáros (2004) and Piran (2005).

2 Originally, Woosley (1993a 1993b) argued against a GRB-SN association and suggested that GRBs are produced by "Failed Supernovae", i.e., by collapsars which do not produce a supernova. After the discovery of the GRB980425/SN1998bw association by Galama et al. (1998), the "Failed Supernovae" became "Hypernovae", i.e. super-energetic supernovae (Iwamoto et al. 1998 MacFadyen \& Woosley 1999). 


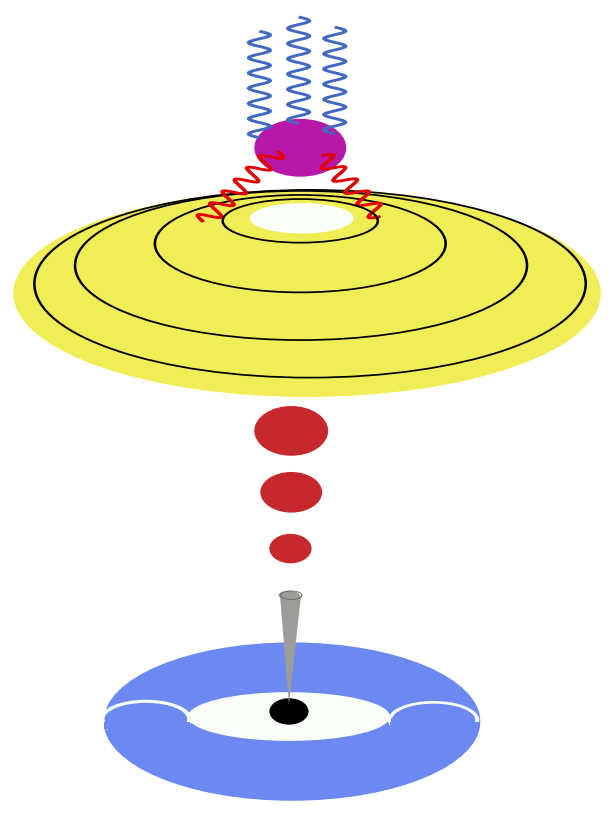

Fig. 2 A fewer-kbyte version of Fig. (3) of Dar \& De Rújula (2000a) showing an "artist's view" (not to scale) of the CB model of GRBs and their afterglows. A corecollapse SN results in a compact object and a fast-rotating torus of non-ejected fallenback material. Matter (not shown) abruptly accreting into the central object produces a narrowly collimated beam of CBs, of which only some of the "northern" ones are depicted. As these CBs move through the "ambient light" surrounding the star, they Compton up-scatter its photons to GRB energies.

the explosion-generating outgoing shock, or by more distant stellar matter falling back after its passage (De Rújula [1987). As observed in microquasars (e.g. Mirabel \& Rodriguez 1999, Rodriguez \& Mirabel 1999 and references therein), each time part of the accretion disk falls abruptly onto the compact object, a pair of CBs made of ordinary atomic matter are emitted with high bulk motion Lorentz factors, $\gamma$, in opposite directions, along the rotation axis, where matter has already fallen back onto the compact object due to lack of rotational support. The $\gamma$-rays of a single pulse in a GRB are produced as a CB coasts through the SN glory - the $\mathrm{SN}$ light scattered by the SN and pre-SN ejecta. The electrons enclosed in the CB Compton up-scatter photons to GRB energies. Each pulse of a GRB corresponds to one CB. The timing sequence of emission of the successive individual pulses (or CBs) in a GRB reflects the chaotic accretion process and its properties are not predictable, but those of the single pulses are (Dar \& De Rújula 2004). An artist's view of the CB model is given in Fig. 2

The rapid expansion of the CBs stops shortly after ejection by their interaction with the ISM (Dado, Dar \& De Rújula 2002). During this rapid expansion and cooling phase their afterglow emission is usually dominated by thermal bremsstrahlung. Later, their emission is dominated by synchrotron radiation from swept-in ISM electrons spiraling in the CBs' enclosed magnetic field. 
Table 1 Main Assumptions of the FB and CB Models of Long Duration GRBs

\begin{tabular}{lcl}
\hline Property & Fireball Model & Cannonball Model \\
\hline Progenitors & Massive Stars, $\mathrm{n}^{*}$-n*/bh Binaries & Massive Stars, Compact Binaries \\
Event & Hypernova, Merger & Supernova, AIC of wd/n* in binary \\
Environment & Progenitor Wind, ISM & SN + Pre SN ejecta, ISM inside SB \\
Remnant & bh & bh, or sq*, or ${ }^{*}$ \\
Ejecta & Baryon poor $e^{+} e^{-}$Shells & Ordinary-Matter Plasmoids \\
Geometry & Conical Shells & Cannonballs (CBs) \\
Ejection time & During Core Collapse & After Fall-Back of Ejecta \\
Radiation & Synchrotron from Colliding Shells & ICS of SN Glory by CBs \\
Lorentz Factor & $\Gamma>300$ & $\Gamma \sim 1000$ \\
Viewing Angle & $\theta<\theta_{j}$ & $\theta \sim 1 / \Gamma$ \\
\hline
\end{tabular}

Abbreviations: $\mathrm{bh}=$ black hole; $\mathrm{n}^{*}=$ neutron star; $\mathrm{sq}^{*}=$ strange quark star; wd = white dwarf; $\mathrm{SN}=$ supernova; $\mathrm{SB}=$ Superbubble; $\mathrm{AIC}=$ accretion induced collapse; $\mathrm{ICS}=$ inverse Compton scattering; $\theta_{j}=$ opening angle of the conical jet (relative to its axis).

Table 2 The FB and CB Model assumptions for AGs of Long Duration GRBs

\begin{tabular}{lcl}
\hline Property & Fireball Model & Cannonball Model \\
\hline Origin & FB Blast Wave in ISM & CB Interaction with ISM \\
Ejecta & Conical $e^{+} e^{-}$Shells & Jet of Ordinary-Matter Plasmoids \\
External Medium & Progenitor Wind, ISM & Progenitor Wind $\rightarrow$ SB $\rightarrow$ ISM \\
"Prompt" AG & SR from Reverse Shock & Brem. from expanding CBs \\
"Normal" AG & SR from Shocked ISM & SR from Decelerating CBs \\
Lightcurve Break & Jet Expansion+Deceleration & Off-Axis Viewing of Decelerating CBs \\
Flares & Extended Central Activity & Encounter with Density Bumps \\
Dark Bursts & Circumburst Absorption & Circumburst Absorption \\
\hline
\end{tabular}

Abbreviations: $\mathrm{SR}=$ Synchrotron Radiation; Brem. $=$ Bremsstrahlung

Table 1 compares the main assumptions of the FB and CB models on the production of GRBs, while Table 2 compares them for AGs.

\section{FB AND CB MODELS CONFRONTING GRB DATA}

Despite their diversity GRBs have a series of common features: The typical energy of their $\gamma$ rays is a fraction of an $\mathrm{MeV}$. The energy distributions are well described by a "Band spectrum", with "peak energies" spanning a surprisingly narrow range. The time structure of a GRB consists of pulses, superimposed or not, rising and decreasing fast. The number of photons in a pulse, the pulses' widths and their total energy vary within broad but specific ranges. Within a pulse, the energy spectrum softens with increasing time. The duration of a pulse decreases at higher energies and its peak intensity shifts to earlier time. Many other correlations between pairs of observables have been identified. Last, based on three measured events, the $\gamma$-ray polarization seems to be very large. A satisfactory theory of GRBs should naturally predict/explain these properties. Table 3 summarizes which GRB properties were correctly predicted/explained by the FB and CB models. Because of space limitation, I shall discuss briefly only a few entries in Table 3 
Table 3 Comparison between Falsifiable Predictions of the FB and CB Models and Observational Data on Long GRBs.

\begin{tabular}{lclc}
\hline Property & Fireball Model & Cannonball Model & Observations \\
\hline "Peak" $\gamma$-Ray Energy & $\mathrm{NP}$ & Eq. (1) & $\sim 250 \mathrm{keV}$ \\
Typical Duration $\left(T_{90}\right)$ & $\mathrm{NP}$ & $\mathrm{NP}$ & $30 \mathrm{~s}$ \\
Mean No of Pulses $\left(n_{p}\right)$ & $\mathrm{NP}$ & $\mathrm{NP}$ & $\sim 6$ \\
Pulse Shape & $\mathrm{FP}$ & Eq⿴囗十 "FRED" & "FRED" \\
"Isotropic Energy"/Pulse & $\mathrm{NP}$ & $0.8 \times 10^{53} \delta_{3}^{3}$ ergs & $10^{48}-10^{54} \mathrm{ergs}$ \\
FWHM of Pulses & $(1+z) R / 2 c \gamma^{2}$ & $\sim 0.5(1+z) / \delta_{3} \mathrm{~s}$ & $1 / 2-200 \mathrm{~s}$ \\
Pulse Spectrum & $\mathrm{Broken} \mathrm{PL}$ & Thermal Brem. + PL Tail & "Band" Spectrum \\
Pulse Spectral Evolution & $\mathrm{NP}$ & Eq. [6 & Hard to Soft \\
Scintillations & $\mathrm{NP}$ & $\mathrm{NP}$ & Debated \\
Polarization & $\mathrm{NP}$ & $2 \theta^{2} \gamma^{2} /\left(1+\theta^{4} \gamma^{4}\right)$ & $>50 \%(3 \mathrm{GRBs})$ \\
Correlations (Pulses): & & & $\sim\left[E_{\gamma}^{i s o}\right]^{0.46 \pm 0.13}$ \\
$(1+z) E_{p}\left(E_{\gamma}^{i s o}\right)$ & $\mathrm{NP}$ & $\sim\left[E_{\gamma}^{i s o}\right]^{1 / 3-1 / 2}$ & $\sim E_{\gamma}^{-1 / 2}$ \\
FWHM $\left(E_{\gamma}\right)$ & $\sim E_{\gamma}^{-1 / 2}$ & $\sim E_{\gamma}^{-1 / 2}$ & $\sim 0.30$ \\
Rise-Time/FWHM & $\mathrm{NP}$ & $\sim 0.27$ & \\
\hline
\end{tabular}

Abbreviations: $\mathrm{NP}=$ Not Predicted; $\mathrm{FP}=$ Failed Prediction; $\mathrm{PL}=$ Power Law; FRED $=$ Fast Rise Exponential Decay; $\delta=\delta_{3} \times 10^{3}$ (Doppler Factor).

3.1 Peak Photon Energy, Isotropic Equivalent Energy and the "Amati Relation" No simple explanation/predictions for the peak photon energy, the isotropic equivalent energy or the "Amati Relation" has been provided by the FB model.

In the CB model (e.g. Dar \& De Rújula 2004), the observed peak-energy of $\gamma$-rays produced at a redshift $z$ by ICS of SN glory with a thermal bremsstrahlung spectrum $d n_{\gamma} / d E \sim$ $E^{-\alpha} \exp (-E / T)$ with a typical energy $\epsilon_{\gamma} \sim T \sim 1 \mathrm{eV}$ and a typical $\alpha \sim 1$, is given by,

$$
E_{p} \approx(2-\alpha) \gamma \delta T /(1+z),
$$

where, $\gamma \sim 1000$ is the initial Lorentz factor of a $\mathrm{CB}, \theta \ll 1$ is the viewing angle relative to the $\mathrm{CB}$ motion and $\delta=1 / \gamma(1-\beta \cos \theta) \approx 2 \gamma /\left(1+\gamma^{2} \theta^{2}\right)$ is its Doppler factor. For the typical viewing angle, $\theta \sim 1 / \gamma$, and the mean redshift, $\langle z\rangle=2.75$ of SWIFT GRBs, $E_{p} \sim 265 \mathrm{keV}$, in agreement with the GRB observations of BATSE, BeppoSAX and SWIFT.

Under the assumption of isotropic emission in the CB rest frame, Doppler boosting and relativistic beaming yield a $\gamma$-ray fluence $F_{\gamma}$ of a GRB pulse, which is proportional to $\gamma \delta^{3}$,

$$
F_{\gamma} \approx \delta^{3}\left[(1+z) E_{\gamma}^{\prime} / 4 \pi D_{L}^{2}\right],
$$

where $E_{\gamma}^{\prime} \sim N_{\gamma} \gamma T$ is the total energy in the CB rest-frame of the $N_{\gamma}$ ambient photons which suffer Compton scattering in the CB. Consequently, under the assumption of isotropic emission in the observer frame, the inferred 'GRB isotropic $\gamma$-ray energy' in a GRB pulse, is

$$
E_{\gamma}^{i s o}=4 \pi D_{L}^{2} F_{\gamma} /(1+z) \approx \delta^{3} E_{\gamma}^{\prime} .
$$

In the $\mathrm{CB}$ model $E_{\gamma}^{\prime} \approx 0.8 \times 10^{44} \mathrm{ergs}$ (Dar \& De Rújula $\left[2004\right.$ ) which yields $E_{\gamma}^{\text {iso }}[$ pulse] $\sim$ $0.8 \times 10^{53}$ ergs for a single pulse, or $E_{\gamma}^{i s o}[G R B] \sim 5 \times 10^{53}$ ergs for the typical values, $\theta \sim 1 / \gamma$ and $\gamma \sim 1000$, and 6 pulses in a GRB, in agreement with observations. 
If core collapse $\mathrm{SNe}$ and their environments were all identical, and if their ejected CBs were also universal in number, mass, Lorentz factor and velocity of expansion, all differences between GRBs would depend only on the observer's position, determined by $z$ and the angle of observation, $\theta$. For a distribution of Lorentz factors that is narrowly peaked around $\gamma \simeq 10^{3}$, the $\theta$-dependence is in practice the dependence on $\delta$, the Doppler factor. Hence Eqs. (11),(3) yield the correlation (Dar \& De Rújula 2000, 2004),

$$
(1+z) E_{p} \propto\left[E_{\gamma}^{i s o}\right]^{1 / 3},
$$

in good agreement with the correlation $(1+z) E_{p} \propto\left[E_{\gamma}^{i s o}\right]^{k}$, with $k=0.35 \pm 0.06$ found by Amati (2004) from an analysis of a sample of 22 GRBs, which were detected and measured with instruments on board BeppoSAX, CGRO and HETE-2, and whose redshift $z$ became available from ground based follow-up optical observations. However, Eq. 目 is marginally consistent with $k=0.40 \pm 0.05$ which was found by Ghirlanda et al. (2004) from a fit to all (40) GRBs and XRFs with known redshift before June 2004. But, GRBs are far from being standard candles and relation (44) is only a crude approximation. For instance, for GRBs with small viewing angle, $\theta^{2} \gamma^{2} \ll 1$, Eqs. (11) and (3) imply $(1+z) E_{p} \propto \gamma^{2}$ and $E_{\gamma}^{\text {iso }} \propto \gamma^{4}$. Then, the spread in $\gamma$ yields $k=0.5$. In the CB model, the expected value of $k$ for the various samples of GRBs and XRFs varies between 0.33 and 0.50. Indeed, the best fitted power-law for $(1+z) E_{p}$ as function of $E_{\gamma}^{i s o}$ for all GRBs/XRFs of known redshift, $E_{p}$ and $E_{\gamma}^{\text {iso }}$, shown in Fig. 3 by a thick line, has $k=0.46 \pm 0.05$ (Dado \& Dar 2005). The parallel thin lines in Fig. 3 ] border the expected spread around the best fit because of the spread in the 'standard candle' properties of GRBs which was found in the CB model (Dar \& De Rújula 2004). As shown in Fig. 3 the correlation predicted by the CB model of GRBs/XRFs is well satisfied, except by GRBs 980425 and 031203 where $E_{p}$ is much larger than expected from their $E_{\gamma}^{i s o}$.

\subsection{Spectrum and Spectral Evolution of GRB Pulses}

In the $\mathrm{CB}$ model, the predicted GRB spectrum from ICS of ambient light with a thermal bremsstrahlung spectrum by the electrons inside the $\mathrm{CB}$, is,

$$
\frac{d N_{\gamma}[1]}{d E} \propto\left(\frac{T_{e f f}}{E}\right)^{\alpha} e^{-E / T_{e f f}}+b\left(1-e^{-E / T_{e f f}}\right)\left(\frac{T_{e f f}}{E}\right)^{\beta},
$$

where $\alpha \approx 1, \beta=(p+2) / 2 \approx 2.1, T_{\text {eff }}=\gamma \delta T /(1+z)$ and $b$ is a dimensionless constant. The values of $\alpha$ and $\beta$ may deviate from their indicated values, because the ambient radiation may deviate from a thin thermal bremsstrahlung, and the power-law index of the accelerated and knocked-on electrons after cooling may be larger than $p+1=3.3$ and increase with time. Also the ambient light temperature seen by the $\mathrm{CB}$ decreases with distance, approximately as

$$
T_{e f f}(t) \sim T_{e f f}(0)\left\{1-\exp \left[-\left(t_{0} / t\right)^{2}\right]\right\},
$$

where $t_{0}$ is a constant. As was shown in Dar \& De Rújula 2004 and in Dado \& Dar 2005, Eq. (5) is practically indistinguishable from the phenomenological Band function (Band et al. (1993) and it is in good agreement with the measured spectrum of the photons in the first peak of the spectral-energy-density of ordinary GRBs and XRFs.

\subsection{Pulse Shape}

The CB model predicts a general shape of GRB pulses,

$$
\frac{d N}{d t}=\exp \left[-\left(\frac{t_{1}}{t}\right)^{m}\right]\left\{1-\exp \left[-\left(\frac{t_{2}}{t}\right)^{n}\right]\right\} .
$$




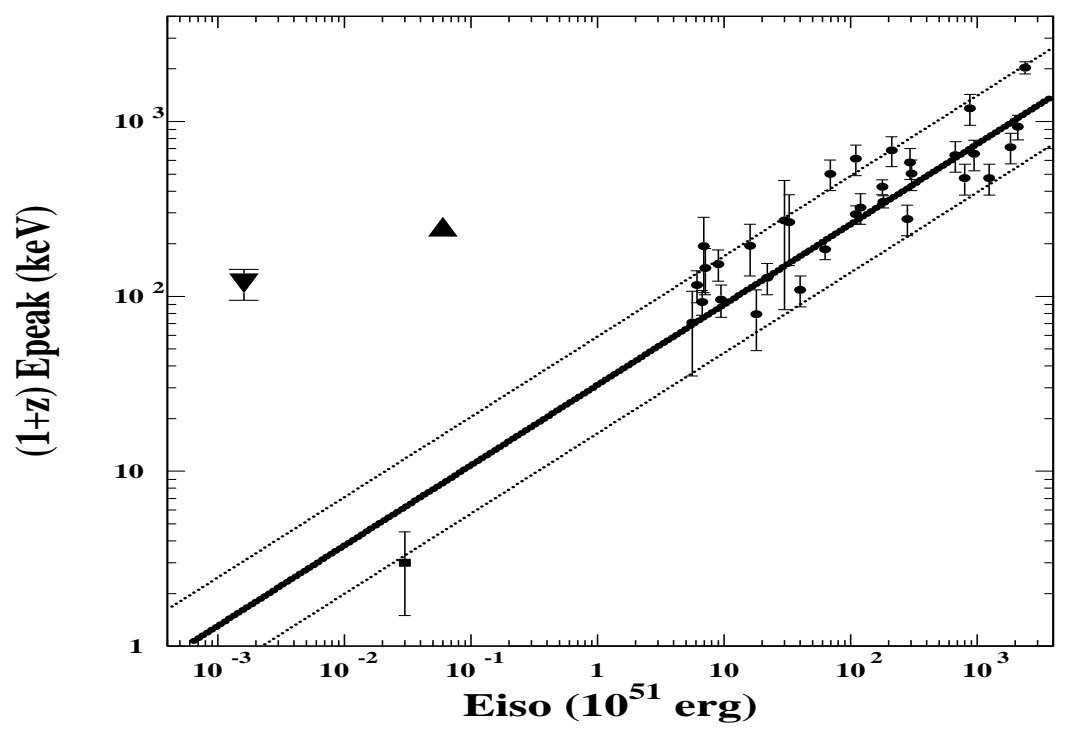

Fig. 3 The observed rest-frame peak-energy as function of the inferred isotropic radiation energy for GRBs/XRFs of known redshift and well measured peak energy. The thick line is the best fitted power-law correlation, $E_{p} \sim\left[E_{\gamma}^{i s o}\right]^{0.46 \pm 0.04}$. The dotted lines border the estimated spread (a factor of $\sim 4$ ) in the isotropic radiation energy due to the spread in $\gamma$ and the angular dependence of the Thomson cross section. The large outlying triangles, which represent $E_{p}$ in GRB 980425 as inferred by Ghirlanda et al. 2004 and a lower limit on $E_{p}$ in GRB 031203 reported by Sazonov et al. (2004), may correspond to a second peak (see Dado \& Dar 2005).

For instance, this pulse-shape fits very well the shapes of the famous single-pulse GRB980425 and the shapes of the two pulses of the famous GRB030329 as demonstrated in Figs. 4 and 5 . Even the most naive pulse-shape, with $m=n=2, t_{1}=t_{2}$, shown in Figs. 4 and [5] does a very good job at describing individual "FRED" shapes as well as results averaged over all observed GRB shapes. For example, for this pulse-shape, the ratio of the rise-time from half-maximum to maximum to the total width at half-maximum is $\simeq 0.27$, while the observed result, reproduced in Fig. [6] is $\sim 0.3$ (Kocevski et al. 2003).

\subsection{Polarization}

The predicted polarization of ambient photons scattered by inverse Compton from the CB electrons into a viewing angle $\theta$ is

$$
\Pi(\theta, \gamma) \approx 2 \theta^{2} \gamma^{2} /\left(1+\theta^{4} \gamma^{4}\right)
$$

which, for the probable viewing angles, $\theta \sim 1 / \gamma$, is naturally large (Shaviv \& Dar 1995 Dar \& De Rújula 2004).

Synchrotron radiation (SR) from a power-law distribution of electrons $d n_{e} / d E \sim E^{-p}$ in a constant magnetic field can produce a large polarization, $\Pi=(p+1) /(p+7 / 3)$, that is $\approx 70 \%$ for a typical $p \approx 2.2$. But a collisionless shock acceleration of the electrons requires highly disordered and time varying magnetic fields (e.g. Zhang \& Meszaros 2004 for a dissenting view 


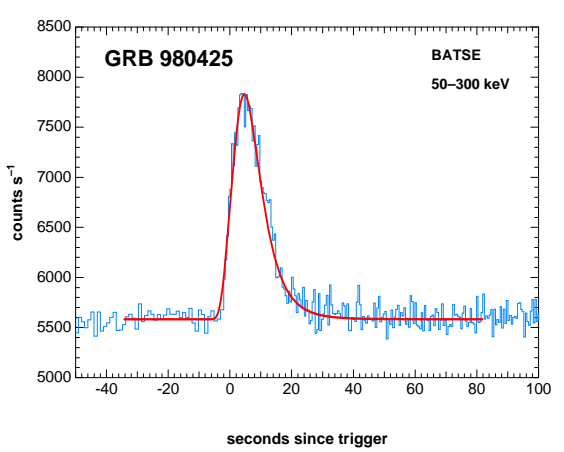

Fig. 4 The light curve $d N_{\gamma} / d t$ of GRB 980425 , as seen by BATSE in the $50-300 \mathrm{keV}$ energy range and its CB model fit (Dar and De Rújula 2004).

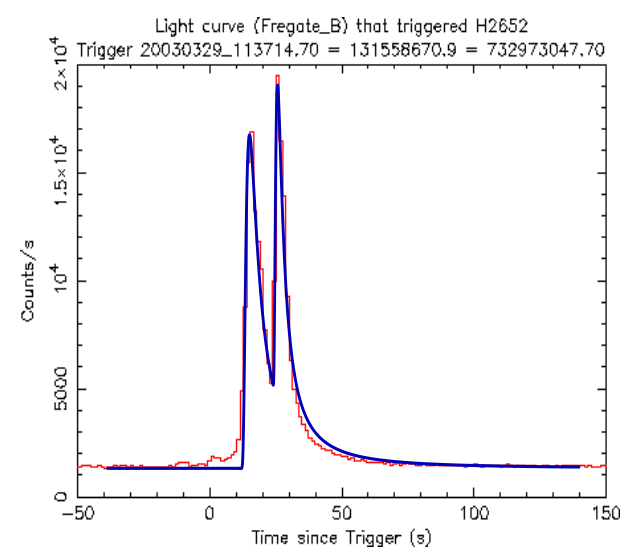

Fig. 5 The lightcurve $d N_{\gamma} / d t$ of GRB 030329, as measured by HETE II showing two dominant pulses and the pulse shapes given by Eq. (7), where only the pulses' heights, widths and relative delay have been adjusted (see Dado et al 2003).

on this point, see Lyutikov, Pariev \& Blandford 2003). Consequently, the expected polarization of GRB and their afterglow is very small (see e.g., Medvedev and Loeb 1999 Lyutikov, Parviev $\&$ Blandford 2003) if the $\gamma$-ray generating mechanism is synchrotron radiation from shockaccelerated electrons moving in highly entangled magnetic field. Indeed, this is the case for the measured polarization of GRB afterglows and the expected GRB polarization in the FB models. But a very large GRB polarization was reported for GRB 021206 (Coburn \& Boggs 2003, see however Wigger et al. 2004) and GRBs 930131 and 960924 (Willis et al. 2005), which clearly advocates the ICS of the CB model, as opposed to the SR of the FB models, as the mechanism generating the $\gamma$-rays of a GRB.

The reported large polarization of GRB 021206 prompted FB model papers on GRB polarization, which showed that under very contrived circumstances - such as geometrical coincidences and unnaturally ordered magnetic fields - shock models of GRBs may also produce a large linear polarization. In our opinion, this is what the articles on GRB polarization by Granot (2003); Eichler \& Levinson (2003); Waxman (2003b); Nakar, Piran \& Waxman (2003) show. Surprisingly, it is not what they say. For instance, Nakar, Piran \& Waxman 2003 reach the opposite conclusion: "the recent detection of very high linear polarization... suggests strongly that these $\gamma$-rays are produced by synchrotron emission of relativistic particles."

\section{FB AND CB MODELS CONFRONT AFTERGLOW DATA}

In both, the FB models and the $\mathrm{CB}$ model, the late-time afterglow of GRBs is produced by synchrotron radiation from a power-law distribution of ISM electrons which were accelerated by the highly relativistic bipolar jets ejected in the SN explosion. However, the geometry and composition of the jet, the deceleration of the jet and the acceleration mechanism of the ISM electrons are different, and result in different predictions and interpretations of the AG data. 


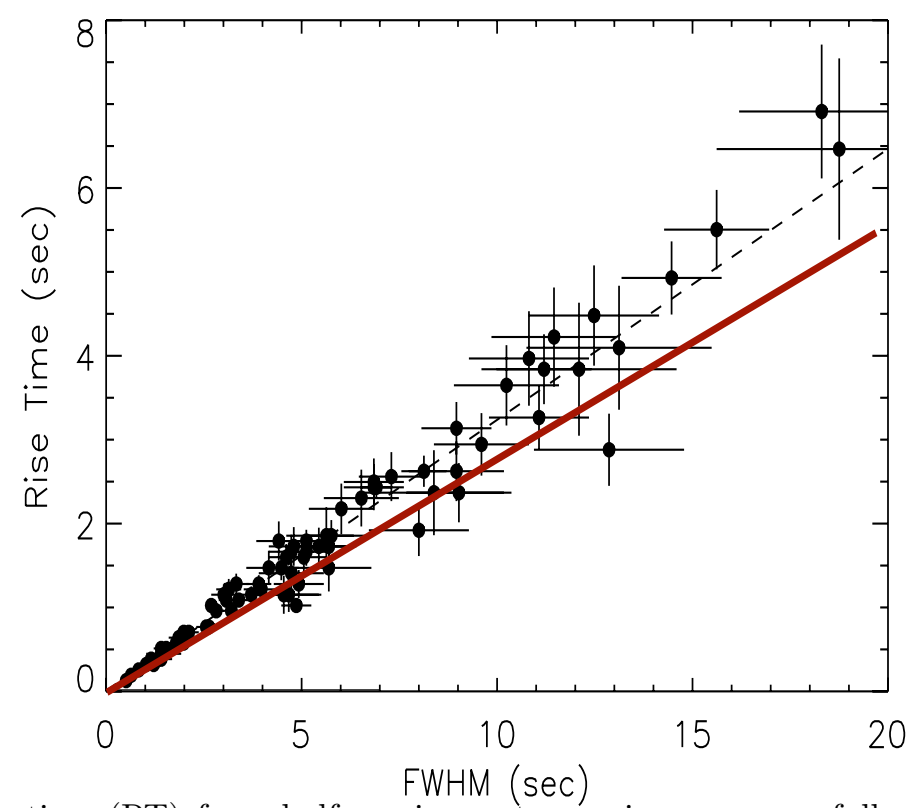

Fig. 6 Rise-time (RT) from half-maximum to maximum versus full width at halfmaximum of an ensemble of GRB single pulses (Kocevski et al. 2003). The data are from pulses of bright BATSE GRBs, the theoretical prediction (the continuous line) is from the naive pulse shape of Eq. (7). The dotted line is the best linear fit, RT $=0.3$ FWHM. The figure has been adopted from Dar \& De Rújula 2004).

Due to space limitation, I shall discuss only 4 key features of the AG phenomena:

\subsection{The Canonical Shape of the Early X-ray Afterglows}

The early X-ray afterglows of GRBs measured by the XRT aboard SWIFT show a universal behaviour: the light curves broadly consist of three distinct power law segments: (i) an initial very steep decay ( $t^{-\alpha}$ with $3<\alpha<5$, followed by (ii) a very shallow decay $(0.2<\alpha<0.8)$, which changes finally to (iii) a steeper decay $(1<\alpha<1.5)$. These power law segments are separated by the corresponding "ankle" and "break" times, 300s $<t_{\text {ankle }}<500$ s and $10^{3} s<$ $t_{\text {break }}<10^{5} \mathrm{~s}$. This is demonstrated in Fig. 8 for GRB 050315 (Vaughan et al. 2005). It was claimed that this universal behaviour cannot easily be explained by current theoretical models of GRBs. This may be true for the popular FB models of GRBs. It is not true, however, for the CB model which predicted this canonical behaviour (Dado et al. 2002). This is demonstrated in Fig. 7 which is borrowed from Dado et al. (2002) where the predicted canonical behaviour was compared with observations of the X-ray AG of several GRBs and in Fig. 8 with the X-ray AG of GRB 050315 observed with the SWIFT XRT (Vaughan et al. 2005).

In the CB model, the energy flux per unit time due to thermal bremsstrahlung (TB) from a CB, after the CB becomes transparent to radiation $\left(t>t_{t r}\right)$, seen by an observer at a luminosity distance $D_{L}(z)$, is given by,

$$
\frac{d F}{d t} \approx \frac{\eta 3 N_{b}^{2} T^{1 / 2} \delta^{4}}{16 \pi^{2} R^{3} D_{L}^{2}} \operatorname{ergs~cm}^{-2} \mathrm{~s}^{-1} .
$$


where $N_{b}$ is its baryon number, $T$ is its plasma temperature in Kelvin, $R$ is its radius in $\mathrm{cm}$ and $\eta=1.473 \times 10^{-27}$. TB dominates the $\mathrm{CB}$ emission for a short (observer) time after the GRB pulse reaches its maximum value at $t \approx t_{t r}$. During this time, $\delta$ does not change significantly, while its radius increases approximately linearly with $t^{\prime}=t-t_{t r}$. The TB emission, which decreases like $R^{-3}$, dominates the $\mathrm{CB}$ emission, until synchrotron emission, which, is proportional to $R^{2}$, takes over. The TB emission by the CB declines like ${ }^{3} R^{-3} T^{1 / 2} \sim t^{\prime-5}$, if its cooling is dominated by the TB emission. If the cooling is dominated by adiabatic expansion, then the emission behaves like $R^{-3} T^{1 / 2} \sim t^{\prime-3.5}$ (Dado et al. 2002).

\subsection{Synchrotron AG from Decelerating Jets}

In the $\mathrm{CB}$ model, the jetted $\mathrm{CBs}$, like those observed in $\mu$-quasars, are assumed to contain a tangled magnetic field in equipartition with the ISM protons which enter it. As the CBs plough through the ISM, they gather and scatter its constituent protons. The re-emitted protons exert an inwards pressure on the CBs which counters their expansion. In the approximation of isotropic re-emission in the CB's rest frame and constant ISM density, $n$, and a constant $\gamma$, one finds that within a few minutes of an observer's time, a CB reaches its asymptotic radius $R$. In the same approximation the deceleration of CBs in the ISM as function of observer time, which depends on the initial $\gamma=\gamma_{0}$ as they become transparent to radiation, on their baryon number $N_{b}$ and radius $R$, on the ISM density $n$ and on their viewing angle, $\theta$, relative to their direction of motion, is given by,

$$
\left[\left(\gamma_{0} / \gamma\right)^{3}-1\right]+3 \theta^{2} \gamma_{0}^{2}\left[\gamma_{0} / \gamma-1\right]=t / t_{0},
$$

where, $t_{0}=(1+z) N_{b} / 8 c n \pi R^{2} \gamma_{0}^{3}$. Hence, $\gamma$ and $\delta$ change very little with time as long as $t<t_{\text {break }}$, where, $t_{\text {break }} \approx\left(1+3 \theta^{2} \gamma_{0}^{2}\right) t_{0}$, i.e.,

$$
t_{\text {break }} \sim 1.8 \times 10^{3}(1+z)\left(1+3 \theta^{2} \gamma_{0}^{2}\right)\left[\frac{\gamma_{0}}{10^{3}}\right]^{-3}\left[\frac{n}{10^{-2} \mathrm{~cm}^{-3}}\right]^{-1}\left[\frac{R}{10^{14}}\right]^{-2}\left[\frac{N_{b}}{10^{50}}\right] \mathrm{s} .
$$

In the $\mathrm{CB}$ model, the electrons that a $\mathrm{CB}$ gathers in its voyage through the ISM are Fermiaccelerated in the $\mathrm{CB}$ enclosed magnetic maze and cooled by synchrotron radiation to a broken power-law distribution with an injection break at the energy $E_{b}=m_{e} c^{2} \gamma(t)$ at which they enter the CB. Their emitted synchrotron radiation has a broken power-law form with a break frequency corresponding to $E_{b}$. In the observer frame, before absorption corrections, it has the approximate form:

$$
F_{\nu} \equiv \nu\left(d n_{\gamma} / d \nu\right) \propto n R^{2}[\gamma(t)]^{3 \alpha-1}[\delta(t)]^{3+\alpha} \nu^{-\alpha},
$$

where $\alpha \approx 0.5$ for $\nu \ll \nu_{b}$ and $\alpha \approx p / 2 \approx 1.1$ for $\nu \gg \nu_{b}$, and

$$
\nu_{b} \simeq 1.87 \times 10^{3}[\gamma(t)]^{3} \delta(t)\left[n_{p} / 10^{-3} \mathrm{~cm}^{-3}\right]^{1 / 2} /(1+z) H z .
$$

The initial slow decline of $\gamma(t)$ and $\delta(t)$ produces the observed shallow decay of the early Xray synchrotron $A G$, provided that the density and the extinction of light from the $\mathrm{CB}$ are constant along the CB trajectory. The sum of TB emission and synchrotron emission from the CB produces the canonical X-ray light curve with an early fast TB decay, which is taken over at the "ankle" by synchrotron emission with an initial shallow decay that rolls over around the "break" to a steeper power-law decline, as demonstrated in Figs. 7 and 8 The shallow decline is not sensitive to the exact deceleration-law of CBs. For instance, if the CBs sweep in the ISM particles and continue to expand according to $R=R_{0}\left(\gamma / \gamma_{0}\right)^{-2 / 3}$ (Dado et al. 2002), then their deceleration-law becomes

$$
\left[\left(\gamma_{0} / \gamma\right)^{8 / 3}-1\right]+4 \theta^{2} \gamma_{0}^{2}\left[\left(\gamma_{0} / \gamma\right)^{2 / 3}-1\right]=8 t / 9 t_{0},
$$




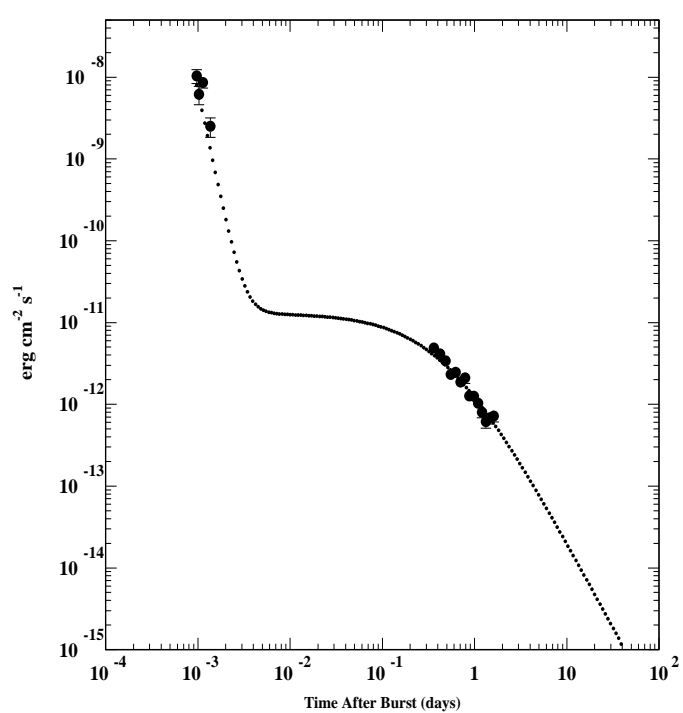

Fig. 7 The universal-shape of the X-ray afterglow of GRBs which was predicted by the CB model in 2002 (Dado et al. 2002) compared with the early and late time X-ray afterglow (2-10 keV) of GRB 990510 as measured by BeppoSAX (Pian et al 2001).

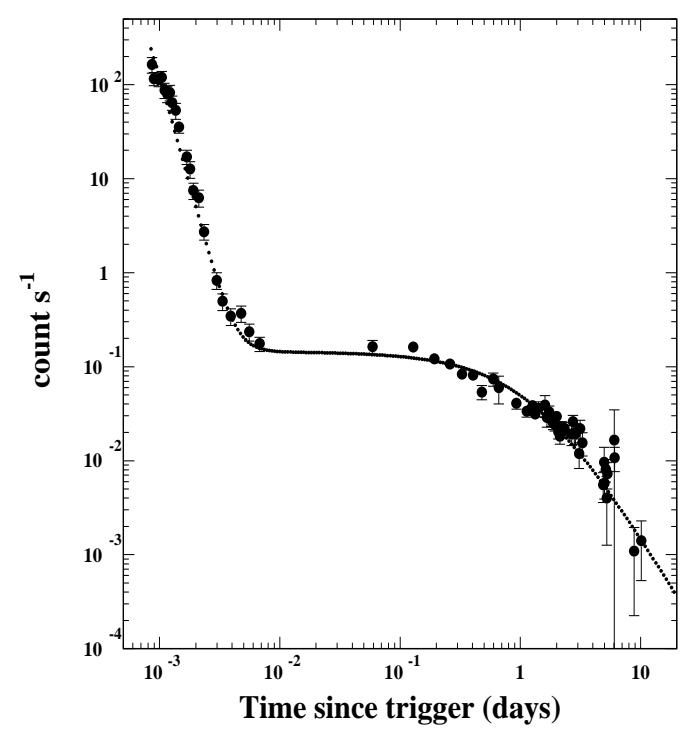

Fig. 8 The X-ray afterglow (0.2-10 keV) of GRB 050315 as measured by the X-Ray Telescope (XRT) on board SWIFT (Vaughan et al. 2005) and a CB model fit (Dado et al. to be published).

and $t_{\text {break }} \sim\left(1+4 \theta^{2} \gamma_{0}^{2}\right)(9 / 8) t_{0}$ is not significantly different from that given by Eq. (11).

\subsection{The AG Break and the Frail relation}

The original blast-wave models assumed that GRBs and their afterglows are produced by spherical fireballs. The 1997 discovery of BeppoSAX that GRBs have afterglows that appear to decline with time like a single power-law was generally accepted as indisputable evidence in support of the model. However, spherical emission implies implausible energy release from small volumes. Repeated claims made by us in print since 1994 (e.g. Shaviv \& Dar 1995), that cosmological GRBs and their afterglows (Dar 1997 1998 Dar \& Plaga 1999) are beamed emissions from highly relativistic jetted ejecta from stellar collapse, were olympically ignored. GRB990123 with its record "equivalent" spherical energy release in $\gamma$ rays was the turning point of the spherical blast wave models. Fireballs became firecones (Rhoads 1999 Sari et al. 1999 Frail et al. 2001) or, more properly, firetrumpets, jets of material funneled in a cone with an initial opening angle (also called $\theta$ ) that increases as the ejecta encounter the ISM. For years these modellers, unaware of the Copernican revolution, placed us, the observers, at a privileged position, precisely on-axis, so that all detected GRBs would point exactly to us. More recently, the FB has evolved towards the realization that the observing angle also matters, a step in the right direction advocated by the CB model: the observation angle is the one that matters.

\footnotetext{
3 The observer time is related to the rest frame CB's time through $d t=(1+z) d t^{\prime} / \delta$, which, for a constant $\delta$ yields the same power-law decline in the CB frame and the observer frame.
} 
Conical FB are claimed to produce a break in the AGs when the beaming angle becomes larger than the opening angle of the conical jet, $\gamma(t)^{-1} \sim \theta_{j}$, and an on-axis observer begins to see the full angular extent of the jet (e.g. Rhoads 1999] Sari et al. 1999). However, sharp breaks were not reproduced by detailed FB calculations that properly took into account arrival time and off-axis viewing effects and an assumed sidewise expansion of the conical jet on top of its ballistic motion, with a speed comparable to the speed of light (Sari et al. (1999). In order to avoid such a difficulties, observers usually use a free broken power-law parametrization (e.g., Stanek et al. 1999 Harrison et al. 1999) of the AG, which is fitted directly to the data rather than a properly derived AGs from the FB model.

In FB models which place the observer on/near the axis of the conical jet, the break time is given by (Sari et al. 1999),

$$
t_{\text {break }} \sim 2.23(1+z)\left[\frac{\theta_{j}}{0.1}\right]^{8 / 3}\left[\frac{n}{0.1 \mathrm{~cm}^{-3}}\right]^{-1 / 3}\left[\frac{\eta_{\gamma}}{0.2}\right]^{-1 / 3}\left[\frac{E_{\gamma}^{\text {iso }}}{10^{53} \text { ergs }}\right]^{1 / 3} \mathrm{day},
$$

where $\eta_{\gamma}$ is the efficiency of the FB in converting the energy of the ejecta into gamma rays and $n$ is the circumburst density. Frail et al. (2001) suggested that the $\gamma$-ray energy of conical GRBs is a standard candle independent of the opening angle of the conical GRB and consequently $E_{\gamma}^{i s o} \approx E_{\gamma} \theta_{j}^{2} / 4$, which became known as the "Frail Relation" 4 . From analysis of 16 GRBs with known redshift, Bloom et al. (2003) found that $E_{\gamma} \approx 1.3 \times 10^{51} \mathrm{ergs}$. The use of this value and the substitution $\theta_{j}^{2}=4 E_{\gamma} / E_{\gamma}^{i s o}$ in Eq. (15), transforms it to,

$$
t_{\text {break }} \sim 4.33(1+z)\left[\frac{n}{0.1 \mathrm{~cm}^{-3}}\right]^{-1 / 3}\left[\frac{\eta_{\gamma}}{0.2}\right]^{-1 / 3}\left[\frac{E_{\gamma}^{\text {iso }}}{10^{53} \mathrm{ergs}}\right]^{-1} \text { day } .
$$

However, all published attempts to use Eq. (16) to predict $t_{\text {break }}$ for AGs of GRBs from the observed $E_{\gamma}^{i s o}$ before it was measured, have failed badly ${ }^{5}$, suggesting that the claimed success of the "Frail relation" may be an artifact and probably because of a posteriori adjustment of free parameters. Moreover, $E_{\gamma}^{\text {iso }}$ for all XRFs with known $z$ seems to be much smaller than the standard candle $E_{\gamma} \approx 1.3 \times 10^{51}$ ergs, implying that XRFs and GRBs cannot be the same phenomenon viewed from different angles, contrary to other indications that they are (e.g. Dado et al. 2004a).

\subsection{AG Bumps and Flares}

The SWIFT XRT provided an unprecedented look at the behaviour of X-ray AG of GRBs in the first few hours after burst. While most of the early AGs have smoothly declining lightcurves, a substantial fraction has large X-ray flares on short time scales (see, e.g Burrows et al. 2005). Late bumps and flares in the AG of GRBs have been detected before in the the X-ray AG of GRB 970508 and also in the optical AG of e.g. GRBs 000301c and 030329 and in the radio $\mathrm{AG}$ of $\mathrm{GRB}$ 030329. In the $\mathrm{CB}$ model, the $\mathrm{AG}$ is a direct and quasi-local tracer of the density of the ISM through which a CB travels: spatial changes in density translate into temporal changes in fluence. In particular, large flares are expected due to CB encounters with density jumps along the CB trajectory (e.g. Dado et al. 2002 2004b). Such density jumps are produced in the circumburst environment by the SN and pre SN ejecta, and by the winds

\footnotetext{
${ }^{4}$ The fraction of the celestial sky which is lighted by a conical GRB is $f_{b}=\left(1-\cos \theta_{j}\right) / 2 \approx \theta_{j}^{2} / 4$ and not $\left(1-\cos \theta_{j}\right) \approx \theta_{j}^{2} / 2$, which was used by Frail et al. (2001) and in many following publications that made use of the "Frail Relation".

${ }^{5}$ For instance, Rhoads et al. 2003 predicted $t_{\text {break }}>10.8$ days for GRB 030226 while shortly after Greiner et al. (2003) reported $t_{\text {break }} \sim 0.8$ day.
} 
in the ISM inside the superbubbles (SBs) where most core collapse SN take place, and in particular, at the complex SB boundaries created by stellar winds and previous supernovae in the SB. This is demonstrated in Figs. 9 and 10

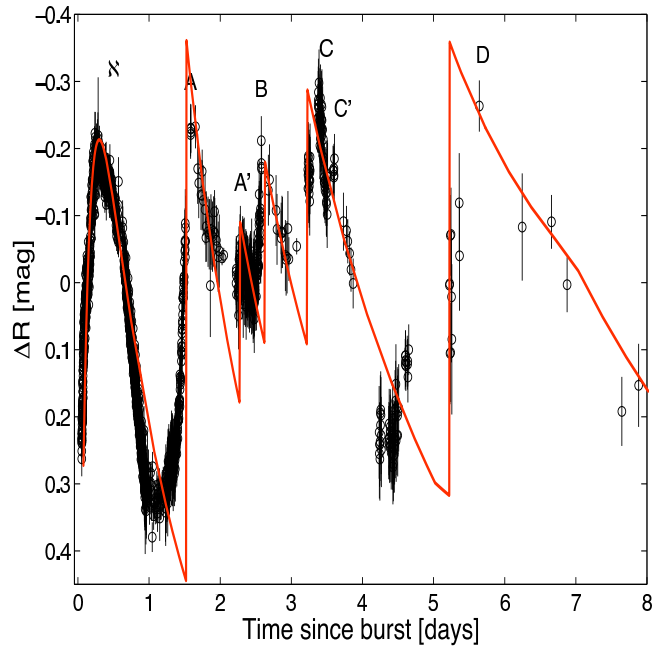

Fig. 9 Comparison between the R-band AG of GRB 030329, shown as "residua" $\Delta R$ of the data (black points and circles) relative to a broken power law of index $-\alpha$ jumping from $\sim 1.1$ to $\sim 2$ at $t \sim 5$ days (Lipkin et al. 2004) and the residua, relative to the same broken power law, calculated from the CB-model (red line) for the input density profile shown on the right hand side (Dado et al. 2004b).

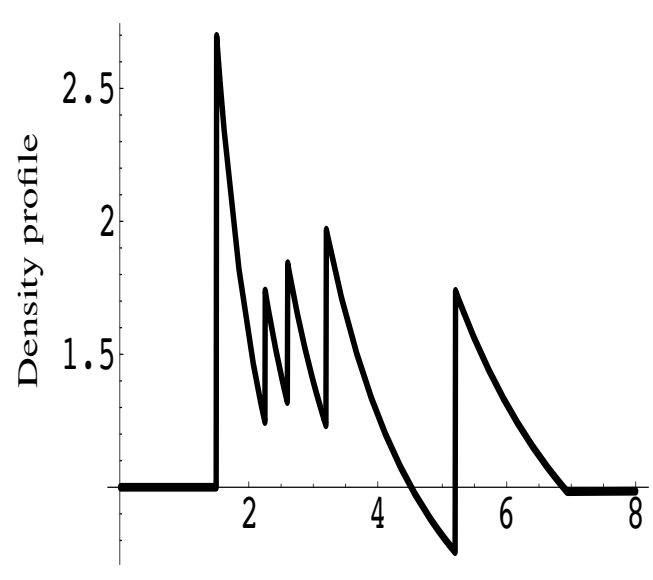

Time in observer's seconds

Fig. 10 The density profile assumed in the CB-model fit to the R-band AG of GRB 030329 (relative to a smooth ISM density - a constant plus a "wind" contribution decreasing as $\left.1 / r^{2}\right)$.

In the FB model, both early and late time flares result from extended central activity (e.g. Granot et al. 2003). Although such an activity can neither be predicted nor ruled out, it is not clear why such late time activity does not produce gamma ray pulses and why the duration and magnitude of the AG flares scale roughly with the time and the magnitude of the declining AG.

\section{CONCLUSIONS}

Confronting the predictions of the FB and CB models with observations of GRBs and their afterglows unmistakenbly demonstrates that the CB model is incredibly more successful than the standard FB or blast-wave models of GRBs, which suffer from profound inadequacies and limited predictive power. The CB model is falsifiable in its hypothesis and results. Its predictions are summarized in simple analytical expressions, derived, in fair approximations, from first principles. It provides a good description on a universal basis of the properties of long-duration GRBs and of their afterglows (AGs). Therefore it is not surprising that the FB models have gradually progressed in the direction of the CB model (compare for instance, Piran 1999 2000 2005): 
- The discovery (Stanek et al. 2003 Hjorth et al 2003) of SN2003dh — associated with GRB 030329 - on the $8^{\text {th }}$ of April, 2003 as predicted a few days earlier by the CB model (Dado et al. 2003), has transformed the GRB-SN association, which underlines the CB model, from a minor and doubtful issue (e.g. Hurley et al. 2002 Waxman 2003a) into something crucial that everybody always knew.

- The angular spread of the ejecta has progressively diminished from an original $4 \pi$ solid angle, to a jet opening angle of tens of degrees (Frail et al. 2001), to $\theta_{j}=10 \mathrm{mrad}$ (Waxman 2003b).

- Following the CB model, the observer's angle, once upon a time set to zero by fiat in the FB model (e.g. Rhoads 1997a, 1999 Sari et al. 1999 Frail et al.2001) is gaining a non-negligible role in FB models (see e.g. Granot et al. 2005] and references therein).

- The correlations discussed in Dar \& De Rújula (2000, 2004) should be approximately valid for any jets seen off-axis. No doubt these correlations will soon be fully exploited as a success of forthcoming off-axis FB models.

- With their current, rather small $\theta_{j}$ values in the conical FB models, GRBs, once, in the era of the spherical FB models, systematically publicized as "the biggest explosions after the Big Bang", have become a small fraction of the SN explosion energy, that is, what they always were in the CB model.

It is not at all inconceivable that the FB models may continue to incorporate and "standardize" other aspects of the $\mathrm{CB}$ model. Four large stumbling blocks lie along this path:

- The traditional GRB-generating mechanism in FB models, synchrotron emission from shock accelerated electrons in entangled magnetic fields, must be replaced by inverse Compton scattering of ambient light, as suggested, for instance, by the very large linear polarization measured in three bright GRBs.

- The scenario of internal and external shocks powering, respectively, the GRB and AG must be forsaken: The observed GRB energy is much larger than the AG energy. Energymomentum conservation implies that the center of mass energy in shell collisions is much smaller than in their collision with the ISM, unless one assumes that most of the bulk motion kinetic energy of the jet is radiated in the internal collisions, which is not supported by observations of relativistic jets in other systems.

- The substance of which the FB-models' ejecta are made: a delicately baryon-loaded (that is, highly fined-tuned) plasma of $e^{+} e^{-}$pairs. Such a fancy substance may not be so difficult to forsake, in comparison with good old ordinary matter.

- The main obstacle may be the magic wand of FB models: shocks.

If and when these obstacles are overcome, the fireballs may turn out to have always been cannonballs for, after all, in the CB model,... SNe fire balls.

One serious drawback of the $\mathrm{CB}$ model is that it makes GRBs become very uninteresting, in comparison with what they used to be: one of the biggest mysteries of astrophysics and the biggest of explosions after the Big Bang. Fortunately, and independently of the "peripheral" GRB- and AG-generating physics, the biggest conundrum remains: How does an SN manage to sprout mighty jets? In the $\mathrm{CB}$ model the guidance along this path is better than simulations: the CBs responsible for GRBs are akin to the increasingly well-studied ejecta of quasars and microquasars presumably fired in hyper-accretion episodes on compact central objects. Moreover, the CB model underlines a unified theory of high energy astrophysical phenomena (e.g De Rújula 2004a) and bridges GRBs to other observational fields as well: cosmic rays (Dar \& Plaga 1999 Dar 20042005 De Rújula 2004b), the gamma background radiation (Dar \& De Rújula 2001a), intergalactic magnetic fields (Dar \& De Rújula 2005) and astrobiology (Dar et al. 1998, Dar \& De Rújula 2001b). 
Acknowledgements This research was supported in part by the Asher Fund for Space Research at the Technion. It is based on an ongoing collaboration with S. Dado and A. De Rújula for which the author is very grateful. S. Vaughan has kindly provided us a tabulated measurememts of the X-ray AG of GRB 050315 with SWIFT XRT.

\section{References}

Amati, L. 2004, arXiv, astro-ph/0405318

Band, D., et al. 1993, ApJ, 413, 281

Bloom, J. S., Frail, D. A. \& Kulkarni, S. R. 2003, ApJ, 594, 674

Burrows, D. N., et al. 2005, arXiv, astro-ph/0511039

Coburn, W. \& Boggs, S. E. 2003, Nature, 423, 415

Dado, S., Dar, A. \& De Rújula, A. 2002, A\&A, 388, 1079

Dado, S., Dar, A. \& De Rújula, A. 2003, A\&A, 401, 243

Dado, S., Dar, A. \& De Rújula, A. 2004a, A\&A, 422, 381

Dado, S., Dar, A. \& De Rújula, A. 2004b, arXiv, astro-ph/0402374

Dado, S. \& Dar, A. 2005, ApJ. 627, L109

Dar, A. 1997, arXiv, astro-ph/9704187

Dar, A. 1998, ApJ, 500, L93

Dar, A. 1999, A\&AS, 138, 505

Dar, A. 2004, arXiv, astro-ph/0408310

Dar, A. 2005, Neutrino Telescopes (ed. M. Baldo-Ceolin) pp. 451-472

Dar, A. \& Plaga, R. 1999, A\&A, 349, 259

Dar, A. \& De Rújula, A. 2000, astro-ph/0008474

Dar, A. \& De Rújula, A. 2001a, MNRAS, 323, 391

Dar, A. \& De Rújula, A. 2001b, arXiv astro-ph/0110162 Astrophysics and Gamma Ray Physics in Space (eds. A. Morselli and P. Picozza), Frascati Physics Series Vol. XXIV pp. 513-523

Dar, A. \& De Rújula, A. 2004, Physics Reports, 405, 203

Dar, A. \& De Rújula, A. 2005, arXiv, astro-ph/0504480

Dar, A., Laor, A. \& Shaviv, N. J. 1998, PRL, 80, 5813

Dermer, C. D. 2002, arXiv, astro-ph/0204037

Dermer, C. \& Mitman, K. 1999, ApJ, 513, L5

De Rújula, A. 1987, Phys. Lett., 193, 514

De Rújula, A. 2004a, arXiv, astro-ph/0411763

De Rújula, A. 2004b, arXiv, hep-ph/0412094

Eichler, D. \& Levinson, A. 2003, ApJ. 596 L147

Frail, D. A., et al. 2001, ApJ, 562, L55.

Galama, T. J., et al. 1998, Nature, 395, 670

Ghirlanda, G., Ghisellini, G. \& Lazzati, D, 2004, ApJ, 616, 331

Ghisellini, G. 2001, arXiv, astro-ph/0101291

Goodman, J. 1986, ApJ, 308, L47

Goodman, J., Dar, A., \& Nussinov, S. 1987, ApJ, 314, L7

Granot, J. 2003, ApJ, 596, L17

Granot, J., Nakar, E. \& Piran, T. 2003, Nature, 426, 138

Granot, J., Ramirez-Ruiz, E. \& Perna, R. 2005, ApJ, 630, 1003

Greiner, J., et al. 2003, GCN circ. 1894 
Harrison, F. A., et al. 1999, ApJ, 523, L121

Hjorth, J., et al. 2003, Natur 423, 847

Hurley, K., Sari, R. \& Djorgovski, S. G. 2002, astro-ph/0211620

Iwamoto, K., et al. 1998, Nature, 395, 672

Katz, J. I. 1994a, ApJ, 422, 248

Katz, J. I. 1994b, ApJ, 432, L107

Kocevski, D., Ryde, F. \& Liang, E. 2003, ApJ. 596, 389

Levinson, A. \& Eichler, D. 1993, ApJ, 418, L386

Lipkin, Y. M., et al. 2004, ApJ, 606, 381

Lyutikov, M., Pariev, V. I. \& Blandford, R. 2003, ApJ, 597, 998

MacFadyen, A. I. \& Woosley, S. E. 1999, ApJ, 524, 262

Medvedev, M. V. \& Loeb, A. 1999, ApJ, 526, 697

Mészáros, P. 2002, ARA\&A, 40, 137

Mészáros, P. \& Rees, M. J. 1992, MNRAS, 257, 29

Mészáros, P. \& Rees, M. J. 1993, ApJ, 405, 278

Mészáros, P. \& Rees, M. J. 1997, ApJ, 476, 232

Mirabel, I. F. \& Rodriguez, L. F. 1999, ARA\&A, 37, 409

Nakar, E., Piran, T. \& Waxman, E. 2003, JCAP, 10, 005

Narayan, R., Paczynski, B. \& Piran, T. 1992, ApJ, 395, L83

Paczynski, B. 1986, ApJ, 308, L43

Paczynski, B. 1998, ApJ, 494, L23

Paczynski, B. \& Rhoads, J. E. 1993, ApJ, 418, L5

Pian, E., et al. 2001, A\&A, 372, 456

Piran, T. 1999, Phys. Rep. 314, 575

Piran, T. 2000, Phys. Rep. 333, 529

Piran, T. 2005, RMP 76, 1143

Rees, M. J. \& Mészáros, P. 1992, MNRAS, 258, 41

Rhoads, J. E. 1997, ApJ, 487, L1

Rhoads, J. E. 1999, ApJ, 525, 737

Rhoads, J. E., et al. 2003, GCN Circ. 1893

Rodriguez, L. F. \& Mirabel, I. F. 1999, ApJ, 511, 398

Sari, R., Piran, T. \& Halpern, J. P., 1999, ApJ, 519, L17

Sazonov, S. Y., Lutovinov, A. A. \& Sunyaev, R. A. 2004, Nature, 430, 646

Shaviv, N. J. \& Dar, A. 1995, ApJ, 447, 863

Shemi, A. \& Piran T. 1990, ApJ, 365, L55

Stanek, K. Z., et al. 1999, ApJ, ApJ, 522, L39

Stanek, K. Z., et al. 2003, ApJ, 591, L17

Vaughan, S., et al. 2005, arXiv, astro-ph/0510677

Waxman, E. 1997a, ApJ, 485, L5

Waxman, E. 1997b, ApJ, 489, L33

Waxman, E. 2003a, Lect. Notes Phys. 598, 393

Waxman, E. 2003b, Nature, 423, 388

Wigger, C., et al. 2004, ApJ, 1, 1

Willis, D. R., et al. 2005, A\&A, 439, 245

Woosley, S. E. 1993, A\&AS, 97, 205 
Woosley, S. E. 1993, ApJ, 405, 273

Yamazaki, R., Ioka, K., Takahara, F., Shibazaki, N. 2005, PASJ, 57, L11, 2005

Zhang, B. \& Mészáros, P. 2004, IJMPA, 19, 2385

This paper was prepared with the ChJAA LATEX macro v1.0. 